Bemerkungen.

Sämmtliche Beobachtungen wurden an dem Steinheilschen Refractor der hiesigen Sternwarte von 5 Zoll Oeffnung und 6 Fuss Brennweite ausgeführt; mit einem Kreismikrometer, dessen Radien $5^{\prime}$ und $8^{\prime}$ sind, und es wurde in der Regel sowohl am inneren wie am äusseren Ringe beobachtet. Vergrösserung etwa $150-180$.

(14) Irene. Juni 28. Luft gut. - Juni 29. Bilder etwas verwaschen und unruhig.

(113) Amalthea. Der Planet ist für dieses Instru. ment schon sehr schwach. - Mai 30 und 3r wurde der Planet bei jedem Durchgange immer gleichzeitig an die beiden Vergleichsterne 3 und 4 angeschlossen. - Mai 30. Luft anfangs befriedigend, später dunstig. - Juni 2. Luft gut, jedoch erscheint Planet sehr schwach. - Juni 3. Luft gut.

Bei den in der Rubrik Vgl. « enthaltenen Gewichtsangaben ist das Gewicht eines vollständigen, sicheren, für beide Gestirne (Stern und Planet) an beiden Ringen ausgeführten Anschlusses $=2$ gesetzt.

In Bezug auf die Reduction der Beobachtungen sei noch erwähnt, dass sie in einem Punkte abweichend von den üblichen Formeln ausgeführt wurde. Die beiden Werthe für den Declinationsunterschied $(\delta-D)$ des Gestirns und des Mikrometercentrums, welche bei jedem einzelnen Durchgange sich aus den beiden Ringen ergeben, wurden nicht zu ein. fachem arithmetischen Mittel, sondern in einer anderen Weise zu einem gemeinsamen Werthe vereinigt: Die Discordanz der beiden Werthe ist durch Zeitfehler der Antritte bedingt; unter der Annahme, dass diese Zeitfehler durch die Licht. schwäche oder Helligkeit des Gestirns (präciser ausgedrückt: durch fehlerhafte Schätzung der halben Intensität) verursacht seien, so dass entweder die Eintritte zu früh und die Austritte zu spät, oder umgekehrt, wahrgenommen wurden, findet man leicht, dass der Zeitfehler am äusseren Ringe sich zu demjenigen am inneren verhalten muss, wie die Cosecanten,

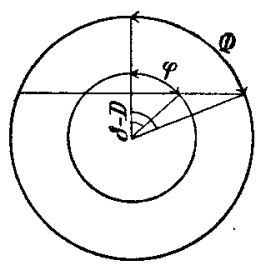
mithin die entsprechenden Correctionen der beiden Werthe für den genannten Declinationsunterschied wie die Secanten der beiden Winkel $\Phi, \varphi$, welche am Centrum durch den Stundenkreis des Centrums und den Radiusvector des äusseren bezw. inneren Antrittspunktes gebildet werden. Man kann so - schon innerhalb des einzelnen Durchganges -

die Zeitfehler desselben eliminiren.

Bei den meisten der beobachteten Durchgänge wurde also der Mittelwerth dieser Bedingung gemäss gebildet. An. gesichts der beträchtlichen Dicke des benutzten Mikrometers war es nicht zulässig, das arithmetische Mittel als einen das so definirte Mittel annähernd darstellenden Werth anzunehmen. Eine Bestätigung der Richtigkeit dieser Mittelbildung kann man wohl in der Thatsache erblicken, dass im Falle der Anwendung des allgemein üblichen Rechnungsverfahrens die verschiedenen Anschlüsse einer Nacht weit schlechter unter einander übereinstimmten, als bei dieser besonderen Mittelbildung. Auch führte keine andere der möglichen Hypothesen, welche über die Natur der Beobachtungsfehler gemacht wurden, - durch entsprechende Elimination dieser Fehler - zu einer so guten Uebereinstimmung der verschiedenen Anschlüsse, wie die oben beschriebene.

München 1898 März.

Lucian Grabozeski.

\title{
Ephemeride des Cometen 1898 I (Perrine März 19).*)
}

Der folgenden für $\mathbf{r}^{\mathrm{b}}$ M. Z. Paris berechneten Ephemeride liegen meine Elemente in A. N. 3499 zu Grunde.

\begin{tabular}{|c|c|c|c|c|c|c|c|c|c|c|c|c|c|c|c|c|c|c|}
\hline \multicolumn{2}{|c|}{1898} & & \multicolumn{3}{|c|}{$\delta$} & \multirow{2}{*}{$\begin{array}{c}\log r \\
0.330 \mathrm{r}\end{array}$} & \multirow{2}{*}{$\frac{\log \Delta}{0.4455}$} & $\mathrm{H}$ & \multicolumn{2}{|c|}{ I 898} & \multicolumn{2}{|c|}{$\alpha$} & \multicolumn{3}{|c|}{$\delta$} & \multirow{2}{*}{$\frac{\log r}{0.3684}$} & \multirow{2}{*}{$\frac{\log \Delta}{0.4589}$} & \multirow{2}{*}{$\frac{\mathrm{H}}{0.07}$} \\
\hline Juli & I 6 & $5^{h} 28^{m} 46^{s}$ & $+53^{\circ}$ & $4^{8}$ & $47 "$ & & & & Aug. & 2 & $5^{b} 5$ & ${ }^{m} 10^{s}$ & $+52^{\circ}$ & $37^{\prime}$ & $15^{\prime \prime}$ & & & \\
\hline & I 7 & 3046 & 53 & 44 & 17 & 3325 & 4466 & 0.08 & & 3 & 55 & 36 & 52 & 33 & 29 & $37 \circ 5$ & 4594 & \\
\hline & 18 & 3244 & 53 & 39 & 48 & 3348 & 4476 & & & 4 & 6 & 0 & $5^{2}$ & 29 & 46 & 3727 & 4599 & \\
\hline & 19 & 3440 & 53 & 35 & $2 \mathrm{I}$ & $337 \mathrm{I}$ & $44^{8} 5$ & & & 5 & & 23 & $5^{2}$ & 26 & 6 & 3748 & 4603 & \\
\hline & 20 & $3^{6} 33$ & 53 & 30 & 55 & 3394 & 4495 & & & 6 & & 44 & 52 & 22 & $3 \mathbf{I}$ & 3769 & 4607 & 0.06 \\
\hline & 2 I & 3825 & 53 & 26 & 32 & 3417 & 4504 & 0.08 & & 7 & & 4 & 52 & 18 & 59 & 3790 & 46 II & \\
\hline & 22 & $40 \quad 14$ & 53 & 22 & I I & $344^{\circ}$ & 45 I 3 & & & 8 & & 21 & 52 & 15 & 31 & $3^{81 \mathrm{I}}$ & 4615 & \\
\hline & 23 & $42 \quad I$ & 53 & 17 & $5^{2}$ & 3463 & $452 \mathrm{I}$ & & & 9 & & 37 & 52 & 12 & 6 & $3^{8} 3^{2}$ & 46 I 8 & \\
\hline & 24 & $43 \quad 4^{6}$ & 53 & I 3 & 35 & $34^{8} 5$ & 4529 & & & 10 & & $5^{I}$ & 52 & 8 & 45 & 3853 & 4621 & 0.06 \\
\hline & 25 & $45 \quad 29$ & 53 & 9 & 22 & 3508 & 4537 & 0.08 & & I I & & 4 & $5^{2}$ & 5 & 29 & 3873 & 4624 & \\
\hline & 26 & $47 \quad 11$ & 53 & 5 & I I & $353^{\circ}$ & 4545 & & & 12 & & 15 & $5^{2}$ & 2 & 17 & $3^{894}$ & 4626 & \\
\hline & 27 & ${ }_{48} \quad 5^{I}$ & 53 & I & 2 & 3553 & $455^{2}$ & & & I 3 & & 23 & $5 \mathrm{I}$ & 59 & 8 & 3914 & 4628 & \\
\hline & 28 & $50 \quad 29$ & $5^{2}$ & $5^{6}$ & $5^{6}$ & 3575 & 4559 & & & I 4 & & 30 & $5 \mathrm{I}$ & $5^{6}$ & 4 & 3934 & $4^{6} 30$ & 0.06 \\
\hline & 29 & $\begin{array}{ll}52 & 4\end{array}$ & 52 & $5^{2}$ & 53 & 3597 & 4566 & 0.07 & & 15 & & 35 & $5 \mathrm{I}$ & 53 & 4 & 3955 & $4^{6} 3^{2}$ & \\
\hline & $3^{\circ}$ & $533^{8}$ & $5^{2}$ & 48 & 54 & 3619 & 4572 & & & 16 & & $3^{8}$ & 51 & $5^{\circ}$ & 8 & 3975 & $4^{6} 33$ & \\
\hline & $3 I$ & 5510 & $5^{2}$ & 44 & $5^{8}$ & 364 I & 4578 & & & I 7 & I & 40 & $5 . \mathrm{I}$ & 47 & 17 & 3995 & 4634 & \\
\hline Aug. & $\mathbf{I}$ & $\begin{array}{lll}5 & 5^{6} & 4^{I}\end{array}$ & $+5^{2}$ & $4 \mathrm{I}$ & 5 & $0.3^{662}$ & $0.45^{8} 3$ & & & I 8 & 61 & 40 & $+5 I$ & 44 & 29 & 0.4015 & $0.4^{6} 35$ & 0.06 \\
\hline
\end{tabular}

*) Eine vom Verfasser eingesandte genaue Ephemeride von Mai I bis Juli I6 kann wegen Raummangels erst in einer der nächsten Nummern zur Veröffentlichung gelangen. $K r$. 


\begin{tabular}{|c|c|c|c|c|c|c|c|c|c|}
\hline \multicolumn{2}{|c|}{1898} & \multicolumn{2}{|r|}{$\alpha$} & \multicolumn{3}{|c|}{$\delta$} & $\log r$ & $\log \Delta$ & $\mathrm{H}$ \\
\hline \multirow{13}{*}{ Aug. } & 19 & $6^{h}$ & $18^{m} 3^{5}$ & $+51^{\circ}$ & $41^{\prime}$ & $46^{\prime \prime}$ & 0.4034 & 0.4635 & \\
\hline & 20 & & 1934 & $5 I$ & 39 & 7 & 4054 & ${ }_{4636}$ & \\
\hline & 2 I & & 2029 & 51 & 36 & 32 & 4074 & 4636 & \\
\hline & 22 & & $21 \quad 22$ & 51 & 34 & 2 & 4093 & $4^{6} 3^{6}$ & 0.05 \\
\hline & 23 & & $22 \quad 13$ & 51 & $3 r$ & 36 & 4112 & 4635 & \\
\hline & 24 & & 232 & 51 & 29 & $r_{4}$ & 4132 & 4634 & \\
\hline & 25 & & 2350 & $5^{1}$ & 26 & $5^{6}$ & $4 I 5 I$ & 4633 & \\
\hline & 26 & & 2435 & 51 & 24 & 43 & 4170 & 4632 & 0.05 \\
\hline & 27 & & $25 \quad 19$ & 51 & 22 & 34 & 4189 & $4^{6} 31$ & \\
\hline & 28 & & $26 \quad 1$ & 51 & 20 & 29 & 4208 & 4629 & \\
\hline & 29 & & 2642 & 51 & 18 & 29 & 4227 & 4627 & \\
\hline & 30 & & 2721 & 51 & I 6 & 32 & 4245 & 4625 & 005 \\
\hline & 31 & & $27 \quad 57$ & 51 & 14 & 40 & 4264 & 4623 & \\
\hline \multirow[t]{9}{*}{ Sept. } & I & & 2831 & 51 & I 2 & 52 & 4283 & 4620 & \\
\hline & 2 & & 294 & 51 & I I & 9 & $430 \mathrm{I}$ & 4618 & \\
\hline & 3 & & 2935 & 51 & 9 & 30 & 4319 & 4615 & 0.05 \\
\hline & 4 & & $30 \quad 4$ & $5^{I}$ & 7 & 54 & $433^{8}$ & 46.11 & \\
\hline & 5 & & $30 \quad 31$ & $5 I$ & 6 & 23 & 4356 & 4608 & \\
\hline & 6 & & $30 \quad 57$ & 51 & 4 & 55 & 4374 & 4605 & \\
\hline & 7 & & 3121 & $5 \mathrm{I}$ & 3 & 32 & 4392 & 4601 & 0.05 \\
\hline & 8 & & 3142 & $5^{I}$ & 2 & 12 & 4410 & 4597 & \\
\hline & 9 & 6 & 322 & $+5 \mathrm{I}$ & 0 & 57 & 0.4428 & 0.4593 & \\
\hline
\end{tabular}

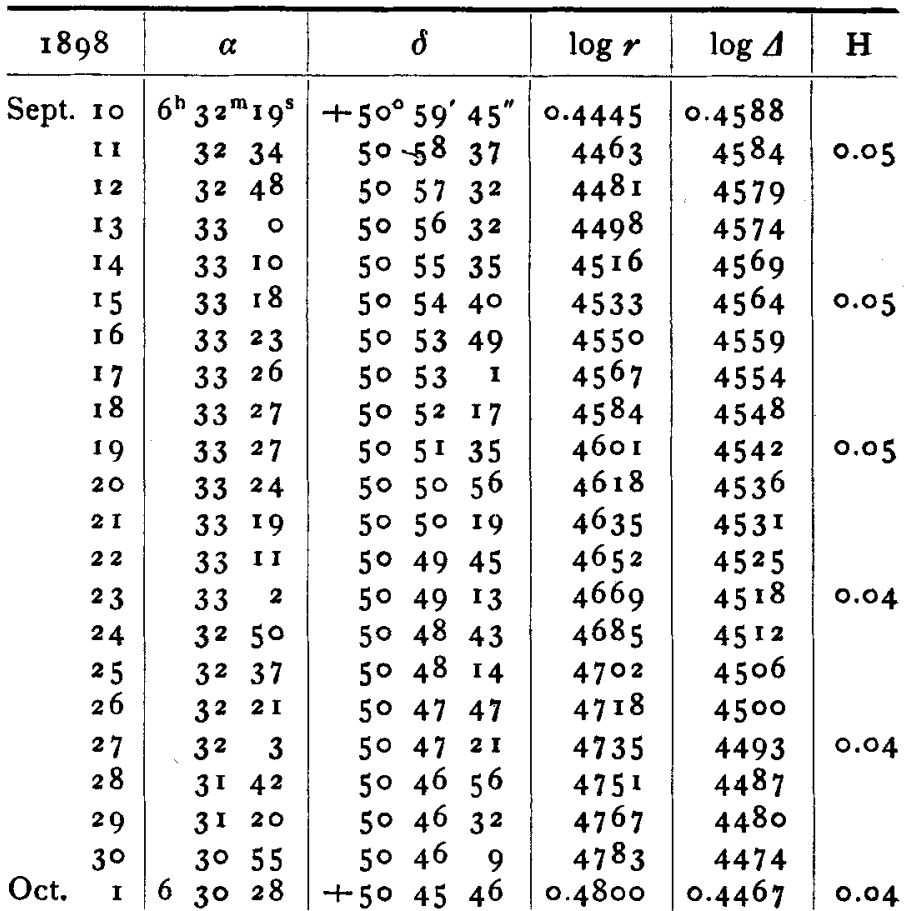

Kopenhagen, I 898 Juni 30 .

S. K. Winther.

Neue Elemente und Ephemeride des Cometen 1898...(Perrine Juni 14).*)

Die Elemente sind berechnet aus den Beobachtungen Darstellung des mittleren Ortes $(B-R)$ :

Mt. Hamilton Juni i 4, Strassburg Juni 20 und Hamburg Juni 23.

$$
\Delta \lambda \cos \beta=+2 " \mathrm{r}, \Delta \beta=+\mathrm{x} \cdot 7 \text {. }
$$

$$
\begin{aligned}
& T=1898 \text { Aug. } 16.3378 \text { M. Z. Berlin } \\
& \omega=204^{\circ} 54^{\prime} 32^{\prime \prime} .9 \\
& \delta=259830.1 \quad \text { I } 898.0 \\
& i=70 \text { I } 7.0 \\
& \log q=9.803019
\end{aligned}
$$

\begin{tabular}{|c|c|c|c|c|c|c|}
\hline \multicolumn{2}{|c|}{1898} & $\alpha$ app. & $\delta$ app. & \multirow{2}{*}{$\frac{\log r}{0.0505}$} & \multirow{2}{*}{$\frac{\log A}{0.253^{\circ}}$} & \multirow{2}{*}{$\frac{\mathrm{H}}{1.78}$} \\
\hline Juli & I & $5^{\mathrm{h}} 5^{\mathrm{m}} 3^{8 \mathrm{~s}}$ & $+52^{\circ} 51: 4$ & & & \\
\hline & $\begin{array}{l}2 \\
3\end{array}$ & $\begin{array}{r}1050 \\
16\end{array}$ & $\begin{array}{ll}5^{2} & 20.4 \\
5 \mathrm{I} & 48.3\end{array}$ & & & \\
\hline & 4 & $21 \quad 18$ & 5115.0 & & & \\
\hline & 5 & 2622 & $50 \quad 40.5$ & 0.0266 & $0.243^{8}$ & 2.08 \\
\hline & 6 & 3122 & $5^{\circ} \quad 4.9$ & & & \\
\hline & 7 & $3^{6} 17$ & $49 \quad 28.1$ & & & \\
\hline & 8 & 418 & $\begin{array}{ll}4^{8} & 50.2\end{array}$ & & & \\
\hline & 9 & $45 \quad 55$ & 48 I I. I & 0.0017 & 0.2347 & 2.43 \\
\hline & Io & 5037 & $47 \quad 30.9$ & & & \\
\hline & II & $\begin{array}{lll}55 & 15\end{array}$ & $46 \quad 49.6$ & & & \\
\hline & I 2 & $\begin{array}{lll}5 & 59 & 49\end{array}$ & $46 \quad 7 \cdot I$ & & & \\
\hline & I 3 & $\begin{array}{lll}6 & 4 & 19\end{array}$ & $45 \quad 23.5$ & 9.9758 & 0.2257 & 2.85 \\
\hline & 14 & 845 & $44 \quad 38.7$ & & & \\
\hline & I 5 & $\begin{array}{lll}6 & 13 & 7\end{array}$ & +4352.7 & & & \\
\hline
\end{tabular}

\begin{tabular}{|c|c|c|c|c|c|c|}
\hline & & $\alpha$ app. & $\delta$ app. & $\log r$ & $\log \Delta$ & $\mathbf{H}$ \\
\hline Juli & I 5 & $6^{\mathrm{h}} \times 3^{\mathrm{m}} 7^{\mathrm{s}}$ & $+43^{\circ} 52: 7$ & & & \\
\hline & 16 & 1725 & $43 \quad 5.6$ & & & \\
\hline & 17 & 2140 & $\begin{array}{ll}42 & 17.3\end{array}$ & $9.949 \mathrm{I}$ & 0.2168 & $3 \cdot 3^{6}$ \\
\hline & 18 & $255^{2}$ & 4127.8 & & & \\
\hline & I9 & 300 & $40 \quad 37.1$ & & & \\
\hline & 20 & $34 \quad 5$ & $39 \quad 45.4$ & & & \\
\hline & $2 \mathrm{I}$ & 38 & $\begin{array}{ll}3^{8} & 5^{2} \cdot 3\end{array}$ & 9.9221 & 0.2080 & 3.96 \\
\hline & 22 & 42 & $37 \quad 58.0$ & & & \\
\hline & 23 & $46 \quad 4$ & $\begin{array}{ll}37 & 2.6\end{array}$ & & & \\
\hline & 24 & 4959 & $3^{6} \quad 5.9$ & & & \\
\hline & 25 & 5351 & 358.1 & 9.8952 & 0.1993 & 4.67 \\
\hline & 26 & $\begin{array}{lll}6 & 5741\end{array}$ & $34 \quad 9.1$ & & & \\
\hline & 27 & $7 \quad 1 \quad 29$ & $33 \quad 8.9$ & & & \\
\hline & 28 & $\begin{array}{ll}5 & 16\end{array}$ & 7.5 & & & \\
\hline & 29 & $\begin{array}{lll}7 & 9 & \text { I }\end{array}$ & +315.0 & 9.8693 & 0.1909 & 5.47 \\
\hline
\end{tabular}

Heliocentrische Aequatorealcoordinaten.

$$
\begin{aligned}
& x=[9.585296] r \cdot \sin \left(v+175^{\circ} 36^{\prime} \quad 8 !^{\prime \prime}\right) \\
& y=[9.999848] r \cdot \sin (v+89 \text { I } 4 \text { 9.4) } \\
& z=[9.965370] r \cdot \sin (v+1795156.2)
\end{aligned}
$$

Ephemeride für $\mathbf{r}^{\mathrm{h}}$ M. Z. Berlin.

Berlin 1898 Juni 29.
A. Berberich.

*) Aus der Beilage zu Nr. 350r wiederholt. 